\title{
Characterization of Spectrum and Eigenvectors of the Schrödinger Operator with Chaotic Potentials*
}

\author{
W.F. OLIVEIRA ${ }^{1}$ and G.Q. PELLEGRINO ${ }^{2 * *}$
}

Received on December 3, 2013 / Accepted on May 28, 2014

\begin{abstract}
Chaotic sequences are sequences generated by chaotic maps. A particle moving in a onedimensional space has its behavior modeled according to the time-independent Schrödinger equation. The tight-binding approximation enables the use of chaotic sequences as the simulation of quantum potentials in the discretized version of the Schrödinger equation. The present work consists of the generation and characterization of spectral curves and eigenvectors of the Schrödinger operator with potentials generated by chaotic sequences, as well as their comparison with the curves generated by periodic, almost periodic and random sequences. This comparison is made by calculating in each case the inverse participation ratio as a function of the system size.
\end{abstract}

Keywords: inverse participation ratio, Schrödinger equation, chaotic sequences.

\section{INTRODUCTION}

One possible way of generating one-dimensional quasi-crystals is the ordering of the atoms in a crystalline lattice by using iteration rules. Some examples of these rules generate Fibonacci, paper-folding, and Thue-Morse sequences, among others (here called almost periodic sequences) $[1,2]$. Although these quasi-crystals are constructed with ordering made from welldefined rules, they do not have a finite period. Because of this fact, they appear as intermediate systems between the periodic and the disordered crystalline structures.

From Bloch Theorem [3], it is known that for periodic potentials the eigensolutions of the Schrödinger operator can be chosen to have periodic absolute values, which enable the particle to move through the entire length of the crystal lattice, leading to good conductors of electricity, heat, etc. On the other hand, and concerning only one-dimensional systems as will be the case

\footnotetext{
*Article presented at Congresso de Matemática Aplicada e Computacional do Centro-Oeste CMAC-CO, Cuiabá-MT, 2013.

**Corresponding author: Giancarlo Queiroz Pellegrino.

${ }^{1}$ Departamento de Ensino, IFNMG - Instituto Federal do Norte de Minas Gerais, Rua Humberto Mallard, 1355, 39900000 Pirapora, MG, Brazil. E-mail: weslley.florentino@ifnmg.edu.br

2 Departamento de Física e Matemática, CEFET-MG - Centro Federal de Educação Tecnológica de Minas Gerais, Av. Amazonas, 7675, 30510-000 Belo Horizonte, MG, Brazil. E-mail: giancarlo@ des.cefetmg.br
} 
here, Anderson Theorem [4] guarantees that disordered potentials confine the particle in finite regions of the lattice, suggesting bad conductors or insulators.

The canonical treatment of the insulator-conductor problem in Solid State Theory uses the tightbinding approximation, where it is assumed that the potential created by the lattice is concentrated in the regions close to the atoms, being negligible in the regions between them [3]. With this approach, one way of solving the one-dimensional time-independent Schrödinger equation takes the tight-binding model of numerical simulation. This in turn considers that an electron moves in a one-dimensional lattice in which each site is occupied by an atom that creates a potential. To this end, sequences of numbers are used to simulate the potential energy for each site.

It is proposed here [5] a new type of sequences, also determined by iteration rules but without definite period, generated from iteration maps more commonly studied in Chaos Theory [6] (hereafter called chaotic sequences).

The present work aims at characterizing the spectrum and eigenfunctions of the one-dimensional Schrödinger operator with potentials generated from chaotic maps. This characterization will be performed by using the definition of the Inverse Participation Ratio [7]. Shortly written, this number provides an estimate of the site contributions to the composition of the wave function and, therefore, gives a measure of its distribution over the lattice. In this context, this characterization should not be taken as a way to determine the spectral type of the Schrödinger operator for the various potentials, but rather as an illustration of the properties shown by the chaotic cases as compared with the others. Nevertheless, such a simple characterization could, we believe, give indications of the possible spectral types.

\section{METHODOLOGY}

In one dimension, the Schrödinger equation is given in the following form:

$$
-\frac{\hbar^{2}}{2 m} \cdot \frac{\partial^{2} \Psi(x, t)}{\partial x^{2}}+U(x) \Psi(x, t)=i \hbar \frac{\partial \Psi(x, t)}{\partial t}
$$

where:

- $\hbar=\frac{h}{2 \pi}$ ( $h$ is the Planck's constant);

- $m$ is the particle mass;

- $t$ is time;

$-x$ is the position of the particle at time $t$;

- $U(x)$ is the potential the particle is subject to at position $x$;

- $i=\sqrt{-1}$;

- $\Psi$ is the (complex) wave function. 
To solve the Schrödinger equation, one can use the method of separation of variables. This method assumes that the function $\Psi(x, t)$ can be written as the product of two others, a timedependent one and a time-independent other.

$$
\Psi(x, t)=\psi(x) \cdot \tau(t)
$$

In this way, two equations are obtained:

1. Time-dependent equation: $\frac{d \tau(t)}{d t}=\frac{E}{i \hbar} \tau(t)$ whose solution is given by $\tau(t)=A e^{\frac{-E t}{i \hbar}}$ where $A$ is a constant to be determined and $E$ is the particle's energy.

2. Time-independent equation: $-\frac{\hbar^{2}}{2 m} \cdot \frac{\partial^{2} \Psi(x, t)}{\partial x^{2}}+U(x) \Psi(x, t)=E \psi(x)$. The solution of this equation depends on the potential to which the particle is subject at the position $x: U(x)$.

In the discrete tight-binding solution method, periodic, aperiodic, and random sequences were used to obtain the solutions of the discretized one-dimensional time-independent Schrödinger equation. We call aperiodic sequences those sequences formed from iteration rules but without finite period, comprising here both almost periodic and chaotic sequences.

Our almost periodic sequences are constructed from substitution rules. As an example, consider a sequence of terms $a$ and $b$, generated by the following rule: $a \rightarrow a b$ and $b \rightarrow a$. Thus, the sequence is being built as follows: $a \rightarrow a b \rightarrow a b a \rightarrow a b a a b \rightarrow a b a a b a b a \rightarrow \cdots$, and successively. This sequence is known as the Fibonacci sequence. Besides this Fibonacci sequence, we took in this work the almost periodic sequences named in the literature as ThueMorse, period-doubling, Rudin-Shapiro, and paper-folding [1, 2] .

The chaotic sequences are obtained from chaotic maps. These maps form sequences iteratively, starting from a chosen initial value. Below, we can observe the formation law for the logistic map:

$$
w_{n+1}=r w_{n}\left(1-w_{n}\right),
$$

where $r \in[0,4]$ is a parameter to be defined and $n$ is the position of the term in the sequence.

The parameter $r$ is decisive for the sequence to become chaotic: as $r$ goes from 0 to 4, the behavior of the logistic sequence becomes chaotic through bifurcations, as shown in Figure 1.

To study the properties of solutions of the Schrödinger operator under chaotic potentials, we sought to raise two important aspects of chaotic maps, which could influence the behavior of those solutions: $(i)$ it is observed that there are different routes to chaos [6]. (ii) the degree of chaoticity can be modified (perhaps in a controlled way) by varying the parameter of the chaotic map. In order to address these issues, we considered also the chaotic maps known as tent map and Gaussian map [6].

One possible method of characterization of the eigenvectors is based on the decay law of $|\psi|^{2}$ as a function of the distance on the lattice taken from its maximum value. In the extreme periodic case, $|\psi|^{2}$ does not decay in a definite way. In the random case, the phenomenon of local- 


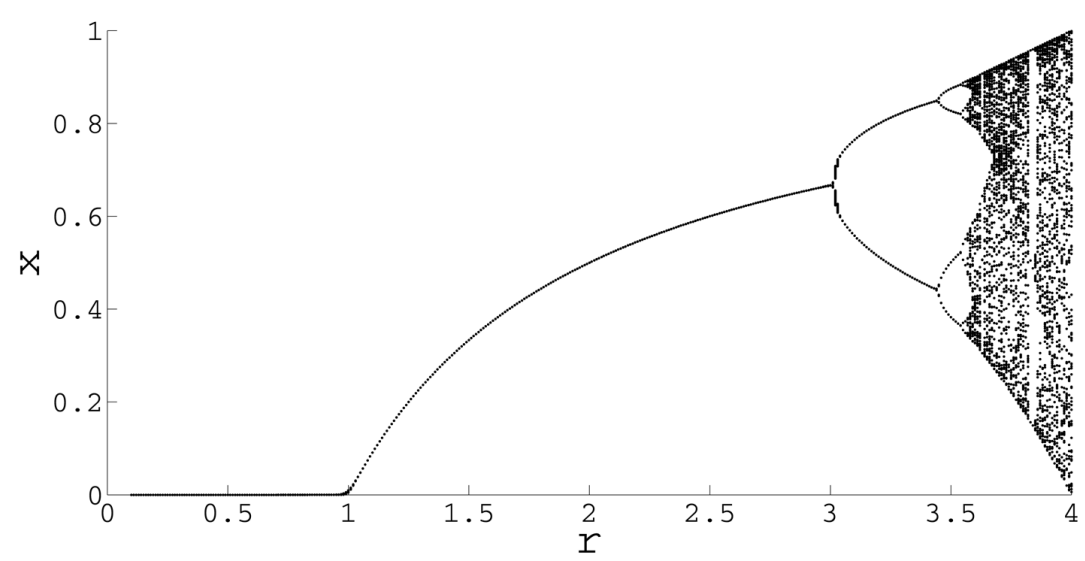

Figure 1: Bifurcation diagram for the logistic map.

ization of $|\psi|^{2}$ is characterized by an exponential decay $e^{-\gamma\left|x-x_{0}\right|}$ with respect to a center $x_{0}$. The intermediate critical cases are given by an algebraic decay of the type $\left|x-x_{0}\right|^{-\alpha}$.

As an alternative to the characterization based on the decay law of $|\psi|^{2}$, we chose to characterize the eigenstates by the decay of the Inverse Participation Ratio ( $I P R)$, a tool closer to Solid State Theory and widely used in studies of the transition localization-delocalization in Anderson models [7]. That quantity is defined for a given wave function as

$$
\operatorname{IPR}(N)=\frac{\sum_{i=1}^{N}\left|\psi_{i}\right|^{4}}{\left(\sum_{i=1}^{N}\left|\psi_{i}\right|^{2}\right)^{2}}
$$

where $\psi_{i}=\psi\left(x_{i}\right)$ is the value of the wave function at site $i$. Defined in this way, this number reflects the contribution of each site to the composition of the wave function and, therefore, its distribution over the lattice.

The algorithm used to numerically solve the Schrödinger equation in the tight-binding method calls as input the sequence to be used and the size of the tridiagonal square matrix related with the problem. From that, it calculates the diagonal matrix of eigenvalues and the eigenvectors associated with each point of the spectrum.

A second algorithm was developed to generate a sequence of IPR's for matrices of increasing sizes $N$, thereby obtaining an $\operatorname{IPR}(N)$ curve. For this curve, a fitting was made in order to obtain its decay law and to analyze it. For the chaotic and random sequences, we chose to analyze the mean curve of IPR sequences, since the behavior of these curves varied with the portion of the sequence used in the tight-binding model.

The following results were obtained by using mean curves constructed from 40 different portions of the sequences used. The curve fitting was done using MATLAB's resources. 


\section{RESULTS}

It is known [7] that for periodic sequences the curve generated by $\operatorname{IPR}(N)$ must decay as $\frac{1}{N}$ for large enough sizes $N$ and hence tend to zero. As for the cases of curves generated by random sequences it should stabilize at a constant value $k>0$. The curves obtained with these two sequences were used as references in the comparison with almost periodic and chaotic sequences, providing in this way a classification with respect to the extreme periodic and random cases known in the literature.

For all curves two fittings were attempted: the first one was exponential and the second one was algebraic. These fittings were suggested by the types of decay characteristic of localized and critical states. From the outset, the exponential fit of the type $a e^{-b x}+c$ was discarded since it presented a low degree of reliability for some sequences. The algebraic fit, in turn, was divided into two types: an algebraic fit of the type $a x^{b}+c$ and a rational algebraic fit of the type $\frac{a x+b}{x+d}$. Note that both admit a behavior of the type $1 / x$ for fitting parameters $b \approx-1$ in the first case and $a \approx 0$ in the second one. Comparing the reliability shown by these two types of algebraic fittings, it can be said that, in general, the algebraic fit of the type $a x^{b}+c$ was the most indicated. In this way, one could obtain the classification table shown in Figure 2.

\section{CONCLUSION}

In general, one can see that the logistic map with parameter $r=3.6$ is the chaotic map whose behavior is closer to the periodic sequence, while the Gaussian map and logistic map with parameter $r=4.0$ compete for better approximating the random sequence. Concerning only logistic maps, as we go deeper into the chaotic region, the behavior tends to be closer to the random case.

The sequences Fibonacci, period-doubling, Thue-Morse, and logistic map with parameter $r=$ 3.6 always positioned themselves close to the periodic sequence, while the Gaussian map, the logistic map with parameter $r=4.0$, and paper-foldind sequence were closer to the random sequence.

A relevant result was the paper-folding sequence to show behavior close to the random one, a result generically opposite to that showed in the characterization method using $|\psi|^{2}$. Moreover, it was also observed that with the logistic map the degree of chaoticity can be controlled. This fact could allows the sequence to be adapted to the specific needs of different physical problems. Therefore, we can conclude that the chaotic sequences can also be used as a way to create the sequenced potentials. It is possible to observe that the chaotic maps enable the generation of curves with characteristics similar to those generated by random or periodic sequences. This suggests materials which are insulators and good conductors, respectively.

In this way, we believe that the chaotic maps may, in parallel with almost periodic sequences, contribute to the study of ordering the atoms and, consequently, to the study of the quasi-crystals and to the engineering of new materials. 


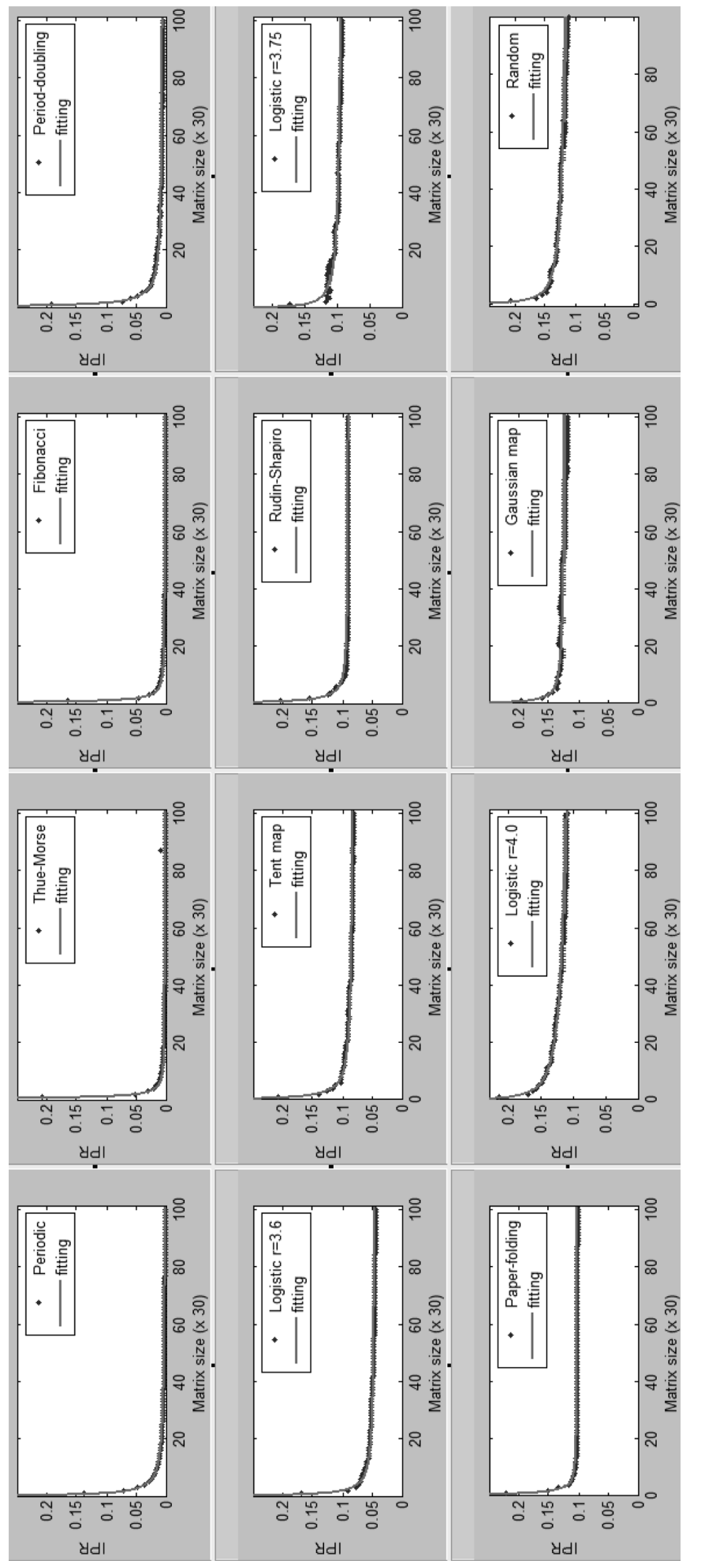

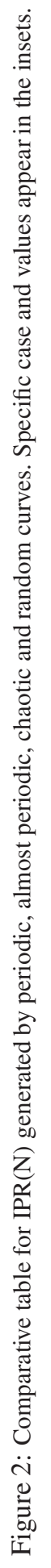


RESUMO. Sequências caóticas são sequências geradas por mapas caóticos. Uma partícula num espaço unidimensional tem seu comportamento modelado segundo a equação de Schrödinger independente do tempo. A aproximação tight-binding possibilita a utilização de sequências caóticas como simulação de potenciais quânticos na versão discretizada da equação de Schrödinger. O presente trabalho consiste na geração e caracterização de curvas espectrais e de autovetores do operador de Schrödinger sob potenciais gerados por sequências caóticas, bem como sua comparação com as curvas geradas por sequências periódica, peneperiódicas e aleatória. Esta comparação é feita calculando-se em cada caso o Inverso do Número de Participação como função do tamanho do sistema.

Palavras-chave: inverso do número de participação, equação de Schrödinger, sequências caóticas.

\section{REFERENCES}

[1] M. Queffélec. "Substitutional Dynamical Systems - Spectral Analysis". Lecture Notes in Mathematics, 1924 (1987), Springer, Berlin.

[2] F. Axel \& D. Gratias (eds.). "Beyond Quasicrystals", Les Editions de Physique, Springer, Berlin (1995).

[3] N.W. Ashcroft \& N.D. Mermin. "Solid State Physics", Rinehart \& Winston, Philadelphia (1976).

[4] P.W. Anderson. Absence of diffusion in certain random lattices. Physical Review, 109 (1958), 1942-1958.

[5] C.R. Oliveira. Private communication (2002).

[6] R.C. Hilborn. "Chaos and Nonlinear Dynamics". Oxford University Press, Oxford (2000).

[7] C. Monthus \& T. Garel. Anderson localization of phonons in dimension $d=1,2$, 3: finite-size properties of the inverse participation ratios of eigenstates. Physical Review B, 81 (2010), 224208-1224208-9. 